\title{
ENERGETICS OF THE DAYSIDE IONOSPHERE OF VENUS
}

\author{
Zoltan Dobe, Andrew F. Nagy, and Larry H. Brace \\ Space Physics Research Laboratory \\ Department of Atmospheric, Oceanic and Space Sciences \\ University of Michigan
}

Thomas E. Cravens

Department of Physics and Astronomy, University of Kansas

Janet G. Luhmann

Institute of Geophysics and Planetary Physics

University of California, Los Angeles

\begin{abstract}
A reanalysis of the Pioneer Venus electron temperature data base showed a strong correlation between elevated electron temperatures and induced magnetic fields in the dayside ionosphere above about $200 \mathrm{~km}$. These results suggest, although not conclusively, that the elevated temperatures are the result of reduced vertical conductivities caused by the horizontal, induced fields with a possible contribution from energy deposition by magnetosheath electrons moving along the field from the tail region.
\end{abstract}

\section{Introduction}

The first indications that calculations based on conventional EUV heating and thermal conduction processes will not lead to the correct daytime plasma temperatures at Mars and Venus came from the Viking 1 and 2 RPA measurements at Mars [Hanson et al., 1977] and associated theoretical model studies [Chen et al., 1978; Johnson, 1978]. Comparisons between the electron and ion temperature measurements, obtained by the various instruments carried aboard the Pioneer Venus Orbiter (PVO) spacecraft [Colin and Hunten, 1977], and model calculations have clearly shown that heat sources beyond that supplied by EUV and/or modified energy transfer processes are important in the ionosphere of Venus (e.g. Cravens et al., 1980; Kim et al., 1990). The one dimensional model calculations of Cravens et al. [1980] demonstrated that temperature values consistent with observations are obtained if either an ad-hoc topside heat inflow into an unmagnetized ionosphere is assumed and/or the electron thermal conductivity is significantly reduced. No direct evidence to justify either of these assumptions has been found, but on the other hand there have been plausible arguments for both of these potential processes.

Taylor et al. [1979] suggested that whistler mode waves are absorbed near the ionopause by the thermal electron population; the energy deposition rate that they predicted was on the same order as needed by the models $\left(\sim 10^{10} \mathrm{eV} \mathrm{cm}^{-2}\right.$ $\mathrm{sec}^{-1}$ ). More recently Szego et al. [1991] proposed that the interaction between the shocked solar wind plasma and ions

\section{Copyright 1993 by the American Geophysical Union.}

Paper number 93GL01452

0094-8534/93/93GL-01452\$03.00 of planetary origin result in the growth of lower hybrid resonance (LHR) waves, which in turn are absorbed in the upper ionosphere, depositing energy on the right order of magnitude ( 4x109 $\mathrm{eV} \mathrm{cm}-2 \mathrm{sec}^{-1}$ ). Magnetic field measurements have clearly demonstrated that at times of relatively high solar wind pressure there are significant induced, horizontal magnetic fields in the ionosphere (e.g. Elphic et al., 1980); even in the absence of these large scale fields small highly fluctuating, even turbulent, fields are present. Both the steady and fluctuating fields result in a significant reduction of the electron thermal conductivity, providing support for the assumption of decreased vertical thermal conductivities in the one dimensional theoretical models. Recently Gan et al. [1990] suggested a modified/new way to explain the observed electron temperatures. They assumed the presence of draped magnetic fields around the dayside ionosphere/ionopause region and showed that EUV heating and electron "precipitation", along the field lines by magnetosheath electrons from the tail region, result in highly elevated temperatures, in agreement with the observed ones.

As mentioned above, the situation at Mars is believed to be similar to that at Venus. However, there is basically no relevant information available at Mars beyond the two vertical profiles obtained by Viking and thus for any hope of progress in this area one needs to concentrate on the Venus data base at the present.

\section{Observations}

Unfortunately no clear observational evidence has been found to date to delineate the processes responsible for establishing the temperature structure in the dayside ionosphere of Venus. Elphic et al. [1984] examined the electron temperature variations in the altitude region of 150 $200 \mathrm{~km}$ to see whether any correlation with the solar EUV fluxes could be established; they did not find such correlation. The ionospheric PVO measurements covered only solar maximum conditions; it was hoped that before entry and burn up in 1992 PVO will be able to add to this data base by making daytime measurements during the declining phase of the solar cycle, but unfortunately it ran out of fuel before it reached the dayside. Luhmann et al. [1987] examined electron and ion temperature, as well as magnetic field, behavior in the Venus ionosphere for different solar wind dynamic conditions; however in this study temperature 
values inside and outside the ionopause were not distinguished.

We decided to take another careful look at the PVO database accumulated during the first three Venus years, when the periapsis was actively controlled, and see if we can learn anything further about the processes controlling the energetics of the daytime ionosphere. More specifically we looked carefully at all available electron temperature data obtained by the Langmuir probe [Brace et al., 1980] from inside the ionosphere. There are numerous "definitions" of the ionopause; in order to make sure that the data that we used was inside the ionosphere, independent of its diverse definition, we restricted the data used to altitudes below the "top of the ionosphere", as defined by Knudsen et al. [1982]. We restricted our study to solar zenith angles between $0-30^{\circ}$, in order to avoid situations where horizontal dynamic flow effects may play an important role; this resulted in restricting our study to only about 45 orbits (we actually also looked at data covering the solar zenith angle range of 30 to $60^{\circ}$ and found the same basic behavior as for the data presented in this paper). We examined each of these orbits in order to classify them as corresponding to "magnetized" or "nonmagnetized" ionospheres. This classification was based on whether there was a significant induced steady magnetic field in the $150-200 \mathrm{~km}$ altitude range. Figure 1 shows the mean altitude profiles of electron temperatures for these two different classes of ionospheric conditions; the standard deviations are indicated by the horizontal bars. In Figure 2 we show the mean magnetic field values corresponding to the two groups of orbits used to obtain Figure 1. The electron temperatures are clearly higher above about $200 \mathrm{~km}$, for the case of magnetized ionospheres. At altitudes below about 200 $\mathrm{km}$ the effects of transport processes are negligible, local equilibrium conditions prevail, therefore it makes "sense" that the temperatures are invariant with respect to the magnetic field. (We solved the coupled electron and ion energy equations to reconfirm that in the presence of no magnetic fields local energy equilibrium dominates below about $200 \mathrm{~km}$ and that heat flow becomes significant above about $220 \mathrm{~km}$.) The data presented in Figures 1 and 2 suggest that the elevated electron temperatures are the result of either 1) the reduction of the heat flow (caused by the reduced

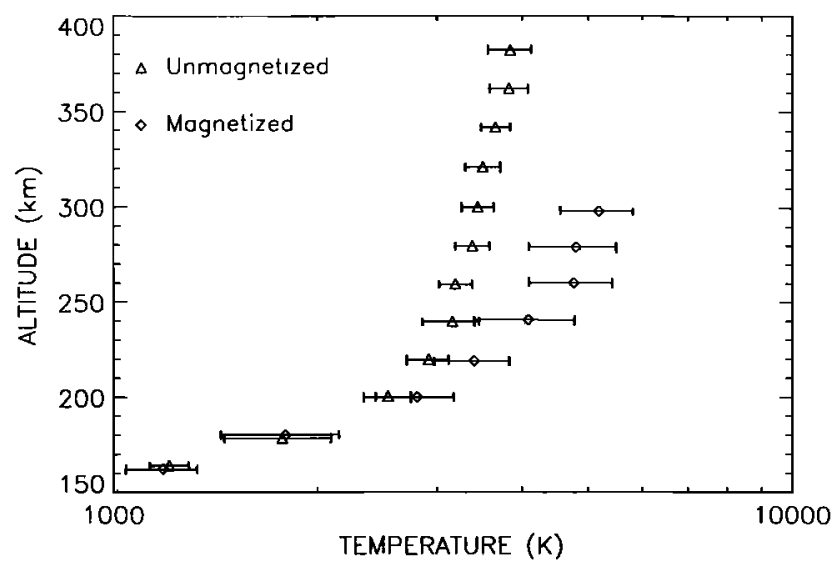

Fig. 1. Average electron temperature values for orbits with SZA between 0 and $30^{\circ}$, grouped according to ionospheric magnetic field conditions (horizontal bars indicate the standard deviations).

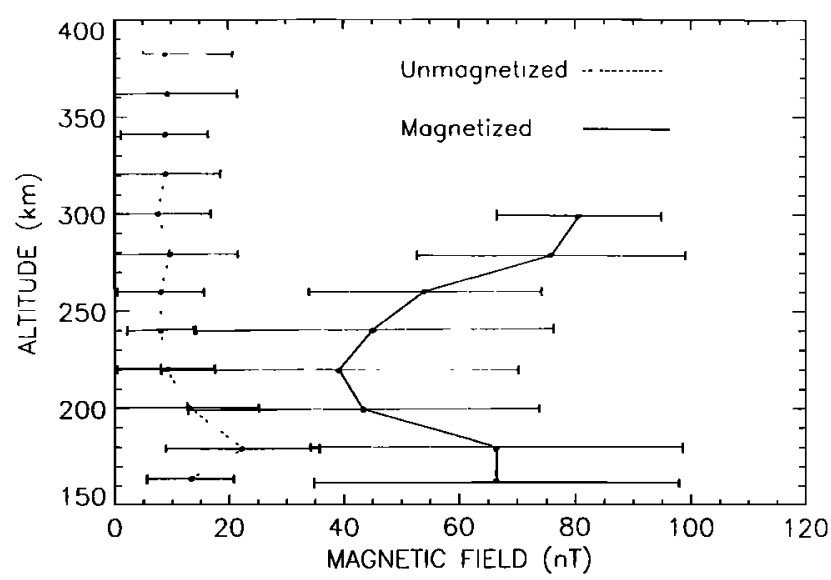

Fig. 2. Average magnetic fields for the two sets of orbits shown in Figure 1. (horizontal bars indicate the standard deviations).

thermal conductivity) from the upper to the lower ionosphere, where collisional losses are high, in the manner proposed by Cravens et al. [1980] and/or 2) the combination of no vertical heat flow and heating by magnetosheath electrons (resulting from the draped field lines) as suggested by Gan et al [1990].

The correlation between the elevated temperatures and the presence of horizontal magnetic field also indicates that topside heat inflows are not the likely cause of these temperatures (see further discussion at the end of this section). As indicated before, horizontal fields cause a major reduction in the thermal conductivity and thus it becomes very difficult to transport heat down from near the ionopause region under these circumstances. It must be pointed out, however, that we cannot state conclusively that the elevated temperatures are the result of the presence of horizontal field, because it has been shown (c.f. Luhmann and Cravens, 1991) that the presence of an induced, mainly horizontal magnetic field, is well correlated with low ionopause altitudes and high solar wind dynamic pressures. The magnitude, efficiency and mechanism of some potential heat source could, in some unknown manner, be related to the solar wind pressure and/or ionopause height, and thus these results could also be indications of some unspecified solar wind ionosphere energy exchange process(es).

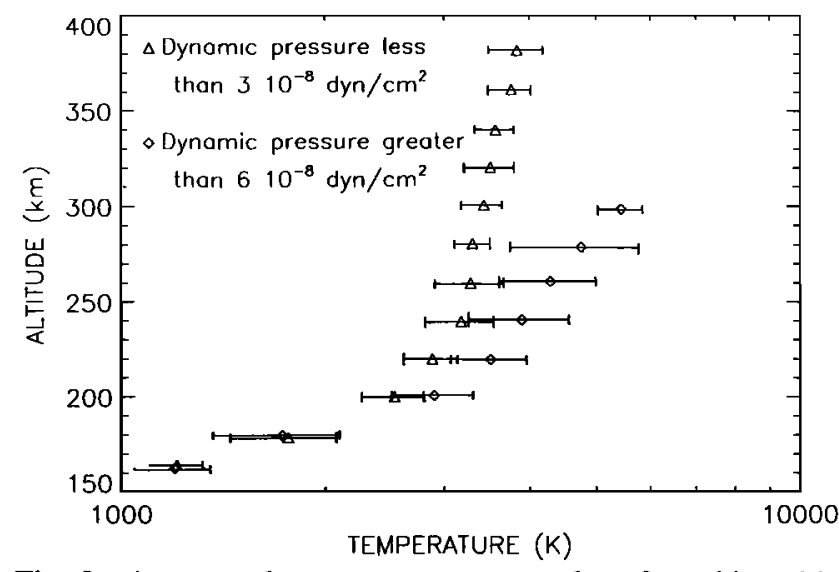

Fig. 3. Average electron temperature values for orbits with SZA between 0 and $30^{\circ}$, grouped according to the unperturbed solar wind dynamic pressure (horizontal bars indicate the standard deviations). 


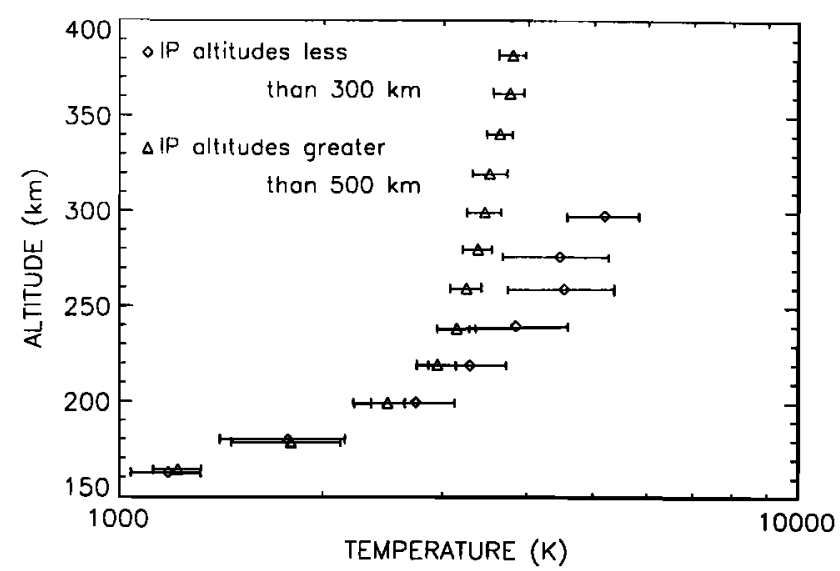

Fig. 4. Average electron temperature values for orbits with SZA between 0 and $30^{\circ}$, grouped according to the ionopause height (horizontal bars indicate the standard deviations).

We also sorted the measured electron temperatures according to the solar wind dynamic pressures, measured outside the bowshock by the Orbiter Plasma Analyzer [J. D. Mihalov, personal communication] and ionopause altitudes; the results are shown in Figures 3 and 4 respectively. As expected and consistent with the results presented in Figure 1 , the electron temperatures are higher corresponding to the high dynamic pressure conditions and low ionopause altitudes.

Solar maximum conditions prevailed during the time period corresponding to our data base, therefore the solar EUV variations were rather small. Nevertheless, we attempted to see if we could establish a meaningful correlation between $T_{e}$ and solar EUV variations. We used the EUV index [Brace et al., 1988] and Figure 5 shows the electron temperatures corresponding to "low" and "high" EUV indices within the time period for which we had data. Our results reconfirm the earlier work of Elphic et al. [1984], which found no meaningful correlation between the electron temperature and the solar flux. Finally we looked at $100 \mathrm{~Hz}$ wave activity and sorted the electron temperatures according to "low" (peak value $<5 \times 10^{-4} \mathrm{~V} / \mathrm{m} \sqrt{\mathrm{Hz}}$ ) and "high" (peak value $>10^{-}$ $3 \mathrm{~V} / \mathrm{m} \sqrt{\mathrm{mHz}}$ ) wave activity conditions prevailing above the

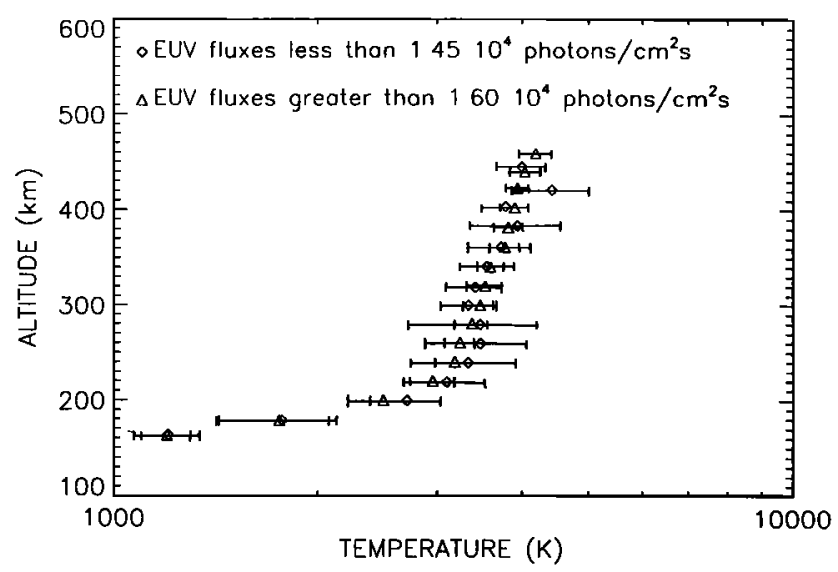

Fig. 5. Average electron temperature values for orbits with SZA between 0 and $30^{\circ}$, grouped according to the solar EUV flux index (horizontal bars indicate the standard deviations).

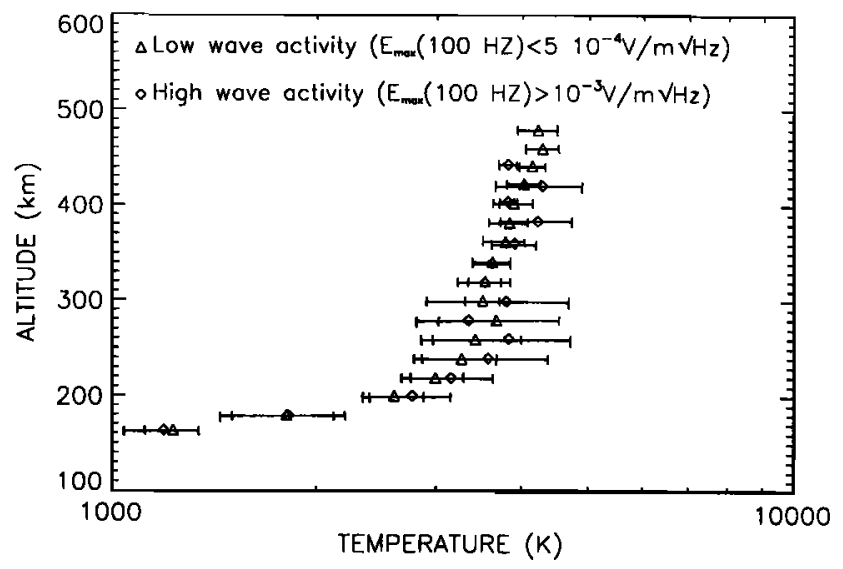

Fig. 6. Average electron temperature values for orbits with SZA between 0 and $30^{\circ}$, grouped according to wave activity (horizontal bars indicate the standard deviations).

ionopause. The results are shown in Figure 6, which show that the observed electron temperatures seem to be independent of this wave activity. The two quantitative suggestions for the source of a topside heat inflow were 1) Landau damping of whistler waves [Taylor et al., 1978] and 2) lower hybrid resonance waves [Szego et al., 1991], both of which are expected to be in the range of $100 \mathrm{~Hz}$. The apparent lack of correlation between the $100 \mathrm{~Hz}$ waves and the electron temperatures reduces the likelihood of the existence of these energy sources.

\section{Discussion}

Our results clearly show that dayside ionospheric electron temperatures are elevated in the presence of an induced ionospheric magnetic field. However this correlation does not prove in a conclusive way that this temperature increase is the result of decreased vertical heat transport due to reduced electron thermal conductivity and/or energy deposition by magnetosheath electrons, because induced fields are also accompanied by increased solar wind dynamic pressure and lowered ionopause heights. However, the most direct conclusion is that the elevated temperatures are the result of the induced horizontal magnetic fields. Furthermore, the lack of correlation of the elevated temperatures with wave activity removes the likelihood that the two quantitative suggestions [Taylor et al., 1979; Szego et al., 1991] for a topside heat source are operative. The definitive resolution of the processes controlling the energetics of the dayside ionosphere cannot be obtained from the existing PVO database. New measurements are needed for a clear answer. The expected similarities between the ionospheres of Mars and Venus may allow progress in this area when results from the upcoming Mars 94 and Planet B missions to Mars become available.

Acknowledgments. The authors thank Dr. W. C. Knudsen for his helpful comments. The work at the University of Michigan was supported by NASA Grants NAG2-491, and NAGW-3579.

\section{References}

Brace, L.H., Hoegy, W.R., and Theis, R.F., Solar EUV measurements at Venus based on photoelectron emission 
from the Pioneer Venus Langmuir probe, J. Geophys. Res., 93, 7282, 1988.

Brace, L.H., Theis, R.F., Hoegy, W.R., Wolfe, J.H., Russell, C.T., Elphic, R.C., and Nagy, A.F., The dynamic behavior of the Venus ionosphere, J. Geophys. Res., 85, 7663, 1980.

Chen, R.H., Cravens, T.E., and Nagy, A.F., The Martian ionosphere in light of the Viking observations, J. Geophys. Res., 83, 3871, 1978.

Colin, L., and Hunten, D.M., Pioneer Venus experiment descriptions., Space Sci. Rev., 20, 451, 1977.

Cravens, T.E., Gombosi, T.I., Kozyra, J.U., and Nagy, A.F., Model calculations of the dayside ionosphere of Venus: Energetics, J. Geophys. Res., 85, 7778, 1980.

Elphic, R.C., Brace, L.H., Theis, R.F., and Russell, C.T., Venus dayside ionospheric conditions: Effects of ionospheric magnetic field and solar EUV flux., Geophys. Res. Lett., 11, 124, 1984.

Elphic, R.C., Russell, C.T., Slavin, J.A., and Brace, L.H., Observations of the dayside ionopause and ionosphere of Venus., J. Geophys. Res., 85, 7679, 1980.

Gan, L., Cravens, T.E., and Horanyi, M., Electrons in the ionospause boundary layer of Venus, J. Geophys. Res., 95, $19023,1990$.

Hanson, W.B., Sanatani, S., and Zuccaro, D.R., The martian ionosphere as observed by the Viking retarding potential analyzer., J. Geophys. Res., 82, 4351, 1977.

Johnson, R.E., Comment on ion and electron temperatures in the martian upper atmosphere., Geophys. Res. Lett., 5, 989, 1978.

Kim, J., Nagy, A.F., Cravens, T.E., and Shinagawa, H., Temperature of individual ion species and heating due to charge exchange in the ionosphere of Venus., $J$. Geophys. Res., 95, 6569, 1990.

Knudsen, W. C., K. L. Miller and K. Spenner, Improved Venus ionopause calculation and comparison with measurement, J. Geophys. Res., 87, 2246, 1982.

Luhmann, J.G., and Cravens, T.E., Magnetic fields in the ionosphere of Venus., Space Sci. Rev., 55, 201, 1991.

Luhmann, J.G., Russell, C.T., Scarf, F.L., Brace, L.H., and Knudsen, W.C., Characteristics of the marslike limit of the Venus-solar wind interaction., J. Geophys. Res., 92, 8545, 1987.

Szego, K., Shapiro, V.S., Shevchenko, V.I., Sagdeev, R.Z., Kasprzak, W.T., and Nagy, A.F., Physical processes in the plasma mantle of Venus., Geophys. Res. Lett., 18, 2305, 1991.

Taylor, W.W.L., Scarf, F.L., Russell, C.T., and Brace, L.H., Absorption of whistler mode waves in the ionosphere of Venus., Science, 205, 112, 1979.

Zoltan Dobe, Andrew F. Nagy, and Larry H. Brace, Space Physics Research Laboratory, Department of Atmospheric, Oceanic and Space Sciences, University of Michigan, Ann Arbor, MI 48109.

Thomas E. Cravens, Department of Physics and Astronomy, University of Kansas, Lawrence, KS 66046.

Janet G. Luhmann, Institute of Geophysics and Planetary Physics, University of California, Los Angeles, Los Angeles, CA 90024.

(Received April 16, 1993; Accepted May 17, 1993.) 